\title{
Úlceras venosas em membros inferiores
}

\section{Venous ulcer in lower extremities}

\section{Johnny Leandro Conduta Borda Aldunate', Cesar Isaac'2, Pedro Ribeiro Soares de Ladeira³, Viviane Fernandes Carvalho ${ }^{4}$, Marcus Castro Ferreira ${ }^{5}$}

\begin{abstract}
Aldunate JLCB, Isaac C, Ladeira PRS, Carvalho VF, Ferreira MC. Úlceras venosas em membros inferiores. Rev Med (São Paulo). 2010 jul.-dez.;89(3/4):158-63.

RESUMO: A úlcera venosa crônica de membros inferiores é extremamente desgastante para o paciente e familiares, deteriorando a qualidade de vida e a produtividade das pessoas. Apesar de ser um problema comum nas unidades de saúde, as úlceras venosas não possuem tratamento adequado na maioria dos serviços devido principalmente ao desconhecimento de sua fisiopatologia e dos métodos terapêticos. Entre as medidas terapêuticas complexas descrita na literatura, a prevenção e o diagnóstico precoce são as etapas primordiais para o tratamento adequado desses pacientes, minimizando as sequelas físicas e psicológicas de uma lesão ulcerada em membros inferiores.
\end{abstract}

DESCRITORES: Úlcera varicosa; Úlcera da perna/fisiopatologia; Ferimentos e lesões/ fisiopatologia; Extremidade inferior/lesões; Cirurgia plástica.

1. Residente de Cirurgia Plástica do Hospital das Clínicas da Faculdade de Medicina da Universidade de São Paulo (FMUSP).

2. Médico membro do Laboratório de Pesquisas em Cultura Celular e Feridas (LIM 04) - Divisão de Cirurgia Plástica Hospital das Clínicas da Faculdade de Medicina da Universidade de São Paulo (HCFMUSP).

3. Acadêmico de Medicina membro do Laboratório de Pesquisas em Cultura Celular e Feridas (LIM 04) - Divisão de Cirurgia Plástica, Hospital das Clínicas da Faculdade de Medicina da Universidade de São Paulo (HCFMUSP).

4. Enfermeira membro do Laboratório de Pesquisas em Cultura Celular e Feridas (LIM 04) - Divisão de Cirurgia Plástica, Hospital das Clínicas da Faculdade de Medicina da Universidade de São Paulo (HCFMUSP).

5. Professor Titular da Disciplina de Cirurgia Plástica da Faculdade de Medicina da Universidade de São Paulo (FMUSP), Diretor da Divisão de Cirurgia Plástica e Queimaduras do Hospital das Clínicas da Faculdade de Medicina da Universidade de São Paulo (HCFMUSP).

Endereço para correspondência: Johny Leandro Conduta Borda Aldunate. Laboratório de Investigação Médica (LIM 04). Av. Dr. Arnaldo, 455 SI. 1363 - São Paulo, SP, Brasil. CEP: 1246-903. 
$\mathrm{L}$ esões ulceradas em membros inferiores podem apresentar diversas etiologias, podendo ocorrer em pacientes com diabetes, insuficiência venosa, insufuciência arterial ou por contato prolongado com superfície riígida. Tais ulcerações tornam-se crônicas quando após um determinado período não apresentam resolução'.

Nos Estados Unidos 500.000 a 1.000 .000 de pessoas sofrem de lesões ulceradas, sendo a maioria de origem venosa'. Ulceras venosas crônicas em membros inferiores afetam mais de 2,5 milhões de pacientes por ano nos EUA, sendo uma causa importante de morbidade e queda na qualidade de vida ${ }^{2}$. Estima-se que o custo do tratamento das ulceras crônicas em membros inferiores por paciente seja de aproximadamente $\$ 30.000$ por ano nos EUA, o que representa $1 \%$ do orçamento da saúde ${ }^{3}$. Além do impacto econômico ocasionado pelas ulceras crônicas em membros inferiores, há também prejuizo na atividade econômica já que tais ulcerações levam a uma perda de 6 milhões de dias de trabalho nos $\mathrm{EUA}^{4}$.

As lesões venosas ulceradas acometem mais frequentemente os indivíduos entre 60 e 80 anos, contudo a maioria das lesões iniciam antes dos 60 anos. Além disso, há um predomínio entre as mulheres, atingindo a proporção de 1,5:1 até 10:15.

\section{Fisiopatologia}

O sistema venoso dos membros inferiores é composto pelas veias superficiais, pelas veias perfurantes e pelas veias profundas. O sistema venoso profuno localiza-se entre a musculatura do membro inferior, enquanto o sistema venosos superficial está acima dessa musculatura. As veias perfurantes são responsáveis pela comunicação entre o sistema venosos profundo e o superficial. Os sistemas venosos descritos são composto por válvulas bicúspedes unidirecionais, promovendo o fluxo sanguíneo apenas para a direção cefálica, prevenindo o refluxo de ssngue ${ }^{4,6}$.

O sangue é direcionado da perna para o coração através da ação de bomba dos músculos da perna. Com a deambulação os músculos da panturrilha contraem, comprimindo o sistema venoso profundo e impulsionando o sangue para a direção cefálica. Com a queda da pressão no sistema venosos profundo, as válvulas fecham prevenindo um fluxo retrógado e o aumento da pressão no sistema venosos superficial. Com o relaxamento da musculatura da panturirilha, há o esvaziamento do sistema venosos profundo e consequentemente uma queda abrupta da pressão, promovendo abertura de válvulas que direcionam o fluxo do sistema superficial ao profundo ${ }^{4,6}$.

Nos pacientes com insuficiência venosa, durante a deambulação, há uma menor queda da pressão no sistema venoso profundo, ocasionando um aumento da pressão no local e consequentemente uma transmissão desse aumento de pressão para o sistema venosos dos membros inferiores ${ }^{4,6,7}$. Sendo assim, a hipertensão venosa é decorrente de uma insuficiência venosa sendo possíveis causas dessa insuficiência uma disfunção valvar no sistema venosos dos membros inferiores que pode ser congênita ou adquirida, disfunção muscular ou falha na "bomba" da musculatura da panturrilha devido a neuropatias, doenças inflamatórias e fibroses ${ }^{8,9}$.

Ainda não é bem esclarecido como a hipertensão venosa leva à ulcerações em membros inferiores ${ }^{10}$, contudo uma dessas teorias descritas por Browse e Burnand relata que a hipertensão venosa provoveria uma distensão da parede dos capilares com extravasamento para a derme e subcutâneo de macromoléculas como o fibrinogênio. No espaço extravascular, esse fibrinogênio é polimerizado formando "cuffs" de fibrina pericapilar que promoveria uma barreira física para a difusão de nutrientes e oxigênio, ocasionando em morte celular e ulceração ${ }^{11}$.

Coleridge-Smith et al. ${ }^{4}$ propõem que com a hipertensão venosa há uma queda no gradiente de pressão entre o sistema arterial e venosos resultando em um baixo fluxo e baixa perfusão nos capilares. Tal alteração promoveria uma agregação de eritrócitos e leucócitos nos capilares, ocasionando uma isquemia local. Há uma liberação de citocinas, enzimas proteoliticas e radicais livres pelos leucócitos que acarretam danos aos vasos levando há uma aumento da permeabilidade vascular e consequentemente há uma perda de macromoléculae como o fibrinogênios.

Falanga e Eaglestein ${ }^{10}$ propuseram uma teoria na qual macromoléculas como a alfa macroglobulina e o fibrinogênio presentes na derme e no subcutâneo após o extravasamento pelos capilares, promoveriam captura de fatores de crescimento e substâncias responsáveis pela homeostase, havendo uma perda da capacidade de manutenção da integridade dos 
tecidos e do processo de reapração ${ }^{4,6,7}$.

\section{Achados clínicos}

As ulceras venosas são classicamente encontradas na "gaiter area" que é a região compreendida entre o tornozelo e a metade da panturrilha e na porção medial da perna, acima do maléolo medial (Figura 1) ${ }^{12}$.

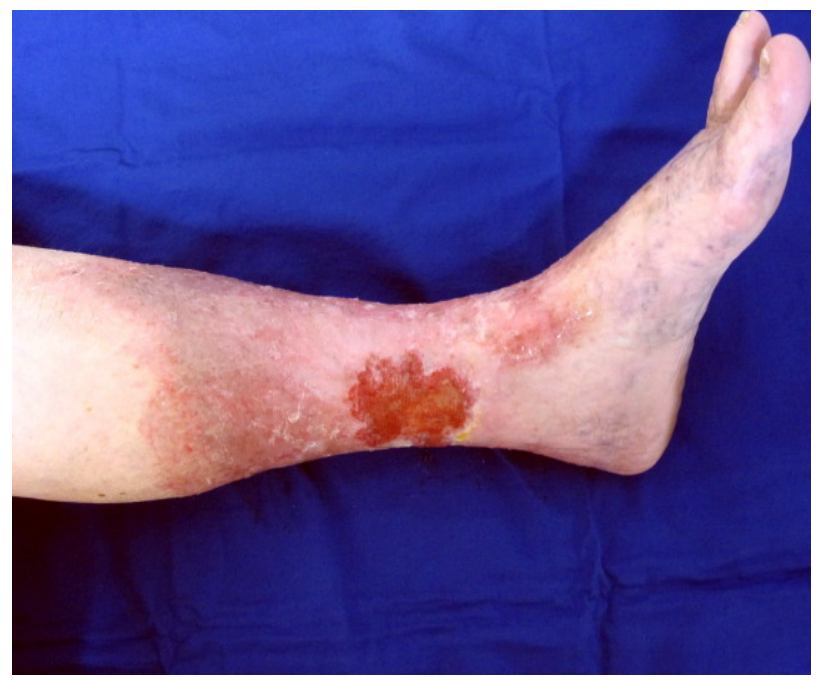

FIGURA 1. Úlcera venosa em porção medial da perna esquerda

O edema em membros inferiores frequentemente está presente, apresentando piora no final do dia e melhora com a elevação do membro 6 . Outro achado presente nos pacientes com ulceras venosas é a hiperpigmentação nos membros inferiores, já que há o extravasamento de eritrocitos para a pele, resultando um depósito de hemosiderina nos macrofagos, estimulando a produção de melanina e pigmentação marrom na pele.

Outra alteração presente nesses pacientes e que normalmente precede a úlcera venosa é a lipodermatoesclerose. Trata-se de uma fibrose crônica da derme e do subcutâneo, ocasionando uma pele firme e endurecida. Em estágios mais tardios, a perna apresenta edema na porção proximal e constrição na distal devido fibrose no e a perda da gordura do subcutâneo, deixando a perna com o formato de uma garrafa de champagne invertida ${ }^{4,13}$.

As úlceras venosas podem ser únicas ou múltiplas, com diversas variações de tamanho, tendendo a serem irregulares e rasas, raramente atingindo músulos, fáscia e ossos ${ }^{4}$.

\section{Tratamento}

O sucesso do tratamento de uma lesão ulcerada está no correto diagnóstico de sua causa. Diversas etiologias estão envolvidas na formação de uma úlcera venosa, sendo que $90 \%$ das úlceras de membros inferiores são decorrentes de insuficiêncoa venosa crônica, insuficiência arterial e neuropatia diabética ${ }^{13}$.

A maiorias das úlceras venosas podem ser diagnosticadas clinicamente ${ }^{14,15}$; já que o paciente apresenta história prévia de problemas venosos tais como veias varicosas, tromboses, coagulopatia, história familiar, dor ou edema ${ }^{11}$. Contudo, testes não invasivos acrescentam a acurácia do diagnóstico, sendo atualemte o ultra som Doppler o método considerado gold standart por causa da sua acurácia, reprodutibilidade e natureza não invasiva. Além disso, esse método também é capaz de avaliar estruras anatomicas na perna que poderiam reproduzir sinais de uma doença venosa, tais como tumores e aneurismas ${ }^{16}$.

Outros testes utilizados são a fotopletismografia e a pletismografia a ar que quantificam os achados do ultra som Doppler. São testes não invasivos que determinam o grau de refluxo venosos e a eficiência da "bomba" da musculatura da panturrilha 4,11,17.

O fator mais importante na prevenção e no tratamento das ulceras venosas é o controle do edema e da hipertensão venosa atraves de medidas compresssivas.

Pacientes submetidos a terapia compressiva apresentam um aumento significativo na taxa de cicatrização e uma queda na recorrência da ulceração. A terapia compressiva ameniza a hipertensão venosa por facilitar o retorno sanguíneo, já que aumenta a função de "bomba" e a drenagem linfática do membro inferior.

Essa melhora do edema local ocorre pelo aumentando da pressão hidrostática e diminuição da pressão venosa superficial, reduzino a perda de fluidos e macromoléculas e aumentando o fluxo sanguineo local ${ }^{18}$. A pressão necessaria para vencer a hipertensão venosa não é conhecida, contudo sabese que pressões acima de 35 a $40 \mathrm{mmHg}$ no tornozelo previne a exsudasão capilar em pernas afetadas por insuficiência venosa ${ }^{11}$.

Há diversas métodos compressivo disponíveis no mercado, divididos em bandagens inelásticas 
e elásticas. A bota de unna é um exemplo de bandagem ineslática utilizado a vários anos para o tratamento da úlcera venosa. Trata-se de uma bandagem rígida e inelástica que promove uma pequena pressão no repouso e uma pressão elevada na deambulação ${ }^{19}$. Apresenta desvantagem de não acompanhar mudanças no tamanho da perna como acontece após diminuição do edema, além disso, necessita de pessoa habilitada na sua confecção e não apresenta uma camada absortiva para acomodar feridas extremamente exsudativas ${ }^{11}$.

Bandagens elásticas incluem as faixas elásticas que são efetivas, devido sua fácil aplicação e a possibilidade de trocas frequentes de curativos. A desvantagem desse método está relacionado com a colocação inadequada da faixa por alguns pacientes e a perda progressiva da elasticidade da faixa após lavagens constantes ${ }^{4}$.

A dificuldade em saber se uma lesão pode ser tratada com medida compressiva sozinha ou em conjunto com terapias adjuvantes é algo que recentemente vem sendo estudado ${ }^{6}$. Estudo retrospectivo apontou alguns fatores de risco para o insucesso da cicatrização da úlcera venosa com apenas medidas compressivas. Entre os fatores descritos estão: longa duração da ulceração, lesões ulceradas de grande tamanho, antecedente de ligaduras venosas e presença de fibrina em mais de $50 \%$ da superfície da ferida ${ }^{6}$.

Uma forma de auxiliar na cicatrização da lesão é a preparação do leito da ferida. É aceito que as lesões ulceradas de membro inferiores devem ser debridadas para permitir um bom tecido de granulação e uma adequada epitelização ${ }^{6}$. Existem diversas formas de debridar uma ferida, existindo métodos químicos, autolíticos e mecânicos que são utilizados asociados com o curativo compressivo. Dentre os métodos de debridamento autolíticos podemos citar o hidrogel, alginatos, películas transparentes e os hidrocolóides ${ }^{6}$.

O debridamento químico é baseado em enzimas debridantes que ao serem colocadas na ferida, promovem a remoção de tecidos necróticos e aceleram a formação do tecido de granulação. Os principais agentes comercializados para esse tipo de debridamento são a colagenase e a papaina ${ }^{6}$.

Quanto ao debridamento mecânico, há diversos métodos de realizá-lo sendo o principal o debridamento cirúrgico. A desvantagem desse método consiste no fato de haver remoção de tecidos viáveis em conjunto com o material necrótico ${ }^{6}$.

O tratamento cirúrgico da ulcera venosa pode ser direcionado para a correção da hipertensão venosa ou ao tratamento propriamente dito da ulceração. Dentre as intervenções cirúrgicas para a causa da hipertensão venosa temos a escleroterapia, a ligadura ou remoção da veia afetada ${ }^{11}$. Não foi confirmado que as intervenções venosas irão resultar em acelereação do processo de cicatrização da lesão, contudo sabe-se que em tais pacientes haverá menor taxa de recorrência da lesão. Sendo assim, parece que até o momento a intervenção cirúrgica para correção do refluxo e da obstrução é essencial apenas para a diminuição da incidência das úlceras venosas $^{12,20,21}$.

Naqueles pacientes com dificuldade de cicatrização da lesão ulcerada, principalmente nas lesões maiores, a enxertia de pele está indicada após adequada preparação do leito da ferida ${ }^{11}$. No Serviço de Cirurgia Plástica do Hospital das Clínicas da Faculdade de Medicina da Universidade de São Paulo (HCFMUSP), há um protocolo para abordagem cirúrgica das úlceras venosas. As lesões ulceradas que não cicatrizam com o tratamento conservador (faixa compressiva associado com curativos) são submetidas, após limpeza com soro fisiológico, a debridamento cirúrgico seguido de colocação de terapia por pressão negativa. A esponja de poliuretano permanece em torno de cinco dias, sendo retirado para avaliação do tecido de granulação. Caso haja granulação de bom aspecto está indicado a enxertia de pele na lesão ulcerada. Um enxerto de pele parcial é retirado conforme as técnica tradicionais e colocado no leito da ferida. Sobre esse enxerto é colocado novo curativo com pressão negativa que permanece por mais cinco dias. Transcorrido esse tempo, o curativo é aberto e avaliado a integração do enxerto (Figura 2).

Após a cicatrização da lesão ulcerada é essencial o acompanhamento adequado dos pacientes para prevenir o retorno das ulcerações. Sabe-se que o uso de meia elástica, cuidados com a pele, manutenção da perna elevada, exercícios com a panturrilha e dieta adequada são medidas importantes e que influênciam para diminuir a chance de retorno das ulceras em membros inferiores ${ }^{12}$. 

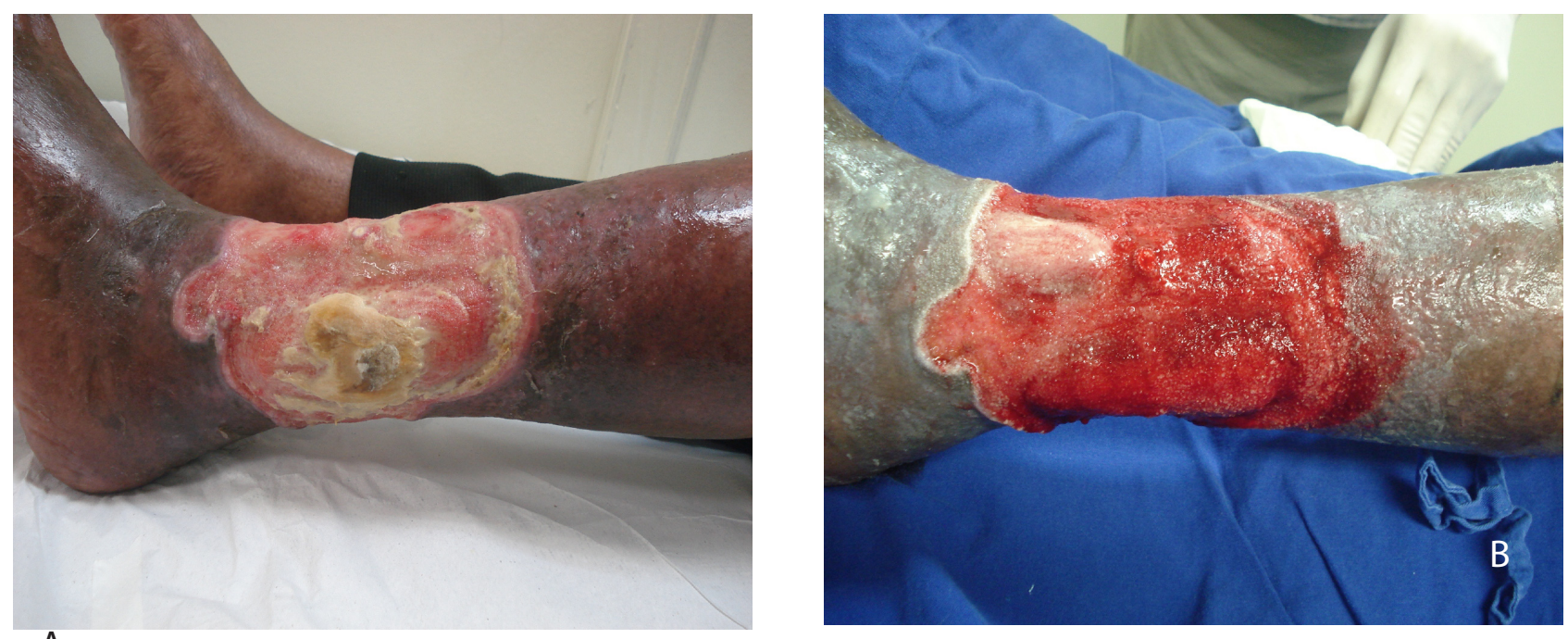

A

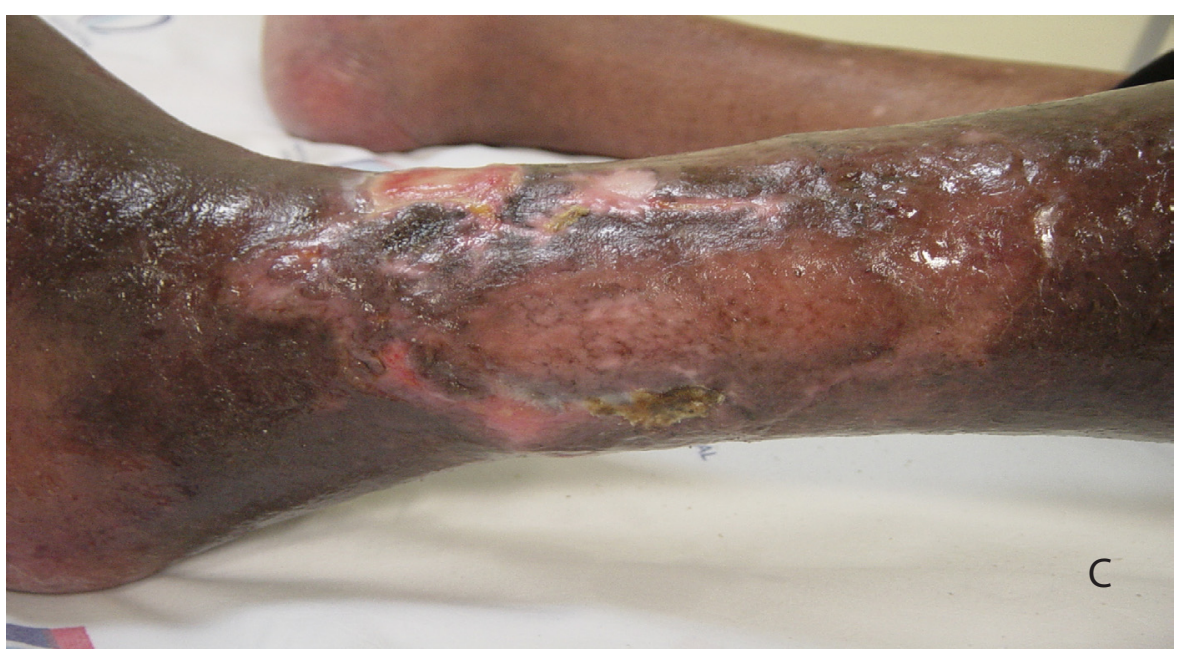

FIGURA 2. Exemplo do protocolo de atendimento de úlceras venosas adotado no serviço de Cirurgia Plástica HCFMUSP. Em (A) observa-se a lesão antes da limpeza cirúrgica, em (B) o aspecto do tecido de granulação após remoção do vacuo e em (C) aspecto após cinco semanas da enxertia de pele

Aldunate JLCB, Isaac C, Ladeira PRS, Carvalho VF, Ferreira MC. Venous ulcer in lower extremities. Rev Med (São Paulo). 2010 jul.-dez.;89(3/4):158-63.

ABSTRACT: The lower limbs chronic venous ulcers are extremely stressful for patients and their families due to the physical limitation and the need of special care caused by that disease, which is responsible for the decrease of their life quality and productivity. Despite being a common problem in health care units, venous ulcers do not have adequate treatment in most of these specialized services mainly because of the ignorance of pathophysiology and therapists. Among the complex therapeutic measures described in the literature, prevention and early diagnosis are the primary steps of a proper treatment; minimizing, thus, the physical and psychological consequences brought to lower limb ulcerations.

KEY WORDS: Varicose; Leg ulcer/physiopathology; Wounds and injuries/physiopathology; Lower extremity/ injuries; Surgery, plastic.

\section{REFERÊNCIAS}

1. Tarlton JF, Bailey AJ, Crawford E, Jones D, Moore K, Harding KD. Prognostic value of markers of collagen remodeling in venous ulcers. Wound Repair Regen. 1999;7(5):347-55. 
2. Mostow EN, Haraway GD, Dalsing M, Hodde JP, King D, OASIS Venus Ulcer Study Group. Effectiveness of an extracellular matrix graft (OASIS Wound Matrix) in the treatment of chronic leg ulcers: a randomized clinical trial. J Vasc Surg. 2005;41(5):837-43.

3. O'Donnell TF Jr, Lau J. A systematic review of randomized controlled trials of wound dressings for chronic venous ulcer. J Vasc Surg. 2006;44(5):111825.

4. Etufugh CN, Phillips TJ. Venous ulcers. Clin Dermatol. 2007;25(1):121-30.

5. Callam MJ. Epidemiology of varicose veins. Br J Surg 1994;81:167-73.

6. Valencia IC, Falabella A, Kirsner RS, Eaglstein WH. Chronic venous insufficiency and venous leg ulceration. J Am Acad Dermatol. 2001;44(3):40121.

7. Raffetto JD, Marston WA. Venous ulcer: what is new? Plast Reconstr Surg. 2011;127(Suppl 1):279S-88S.

8. Rudolph D. Standards of care for venous leg ulcers: compression therapy and moist wound healing. J Vasc Nurs. 2001;19(1):20-7.

9. Gourdin FW, Smith JG. Etiology of venous ulceration. South Med J. 1993;86:1142-6.

10. Falanga V, Eaglestein W. The trap hypothesis of venous ulceration. Lancet. 1993;341:1006-8.

11. Patel NP, Labropoulos N, Pappas PJ. Current management of venous ulceration. Plast Reconstr Surg. 2006;117(7 Suppl):254S-260S.

12. Grey JE, Harding KG, Enoch S. Venous and arterial leg ulcers. BMJ. 2006;11;332(7537):347-50.

13. Marston W. Evaluation and treatment of leg ulcers associated with chronic venous insufficiency. Clin Plast Surg. 2007;34(4):717-30.

14. Scriven JM, Hartshorne T, Bell PR, Naylor AR, London NJ. Single-visit venous ulcer assessment clinic: the first year. Br J Surg. 1997;84:334-6.

15. Lopez A, Phillips TJ. Venous ulcers. Wounds. 1998;10:149-57.

16. Buchbinder D, McCullough GM, Melick CF. Patients evaluated for venous disease may have other pathological conditions contributing to symptomatology. Am J Surg. 1993;166:211-5.

17. Labropoulos N, Leon M, Geroulakos G, Volteas N, Chan $\mathrm{P}$, Nicolaides AN. Venous hemodynamic abnormalities in patients with leg ulceration. Am J Surg. 1995;169:72-4.

18. Partsch H. Compression therapy of the legs: a review. J Dermatol Surg Oncol. 1991;17:799-808.

19. Valencia IC, Falabella A, Kirsner R, Eaglstein W. Chronic venous insufficiency and venous leg ulceration. J Am Acad Dermatol. 2001;44:401-2.

20. Gillespie DL, Writing Group III of the Pacific Vascular Symposium 6, Kistner B, Glass C, Bailey B, Chopra A, et al. Venous ulcer diagnosis, treatment, and prevention of recurrences. J Vasc Surg. 2010;52(5 Suppl):8S$14 \mathrm{~S}$.

21. Douglas WS, Simpson NB. Guidelines for the management of chronic venous leg ulceration: report of a multidisciplinary workshop. Br J Dermatol. 1995;132:44652. 